\title{
Comparison of a Continuous Noninvasive Temperature to Monitor Core Temperature Measures During Targeted Temperature Management
}

\author{
Melissa Wagner ${ }^{{ }^{*}} \mathbb{0}$, Krista Lim-Hing ${ }^{2}$, Mary Ann Bautista ${ }^{3}$, Brigid Blaber ${ }^{3}$, Taghi Ryder ${ }^{3}$, Joseph Haymore ${ }^{4}$ \\ and Neeraj Badjatia ${ }^{5}$
}

C 2020 Springer Science+Business Media, LLC, part of Springer Nature and Neurocritical Care Society

\begin{abstract}
Background: Temperature modulating devices (TMD) currently utilize core temperature measurements during targeted temperature management (TTM) that are currently limited to esophageal (Et), bladder (Bt), or rectal (Rt) temperatures. We assessed the ability of a continuous noninvasive temperature monitor to accurately approximate core temperature during TTM.
\end{abstract}

Methods: All patients undergoing TTM using a gel pad surface TMD and an existing core temperature monitoring device were eligible for this study. Core and continuous noninvasive temperature monitoring values were simultaneously recorded for up to $72 \mathrm{~h}$ of TTM. The two sets of temperature data were downloaded from a clinical data acquisition storage system at 1-min intervals. The Bland-Altman method assessed agreement between the core and continuous noninvasive temperature monitor values, by measuring the mean difference ( $\pm 2 \mathrm{SD}$ ) between these values.

Results: There were 20 subjects that underwent study between January 2018 and March 2018 (55\% women, age: $57 \pm 14$ years old, BMl: $28.9+9.8 \mathrm{~kg} / \mathrm{m}^{2}, 100 \%$ mechanically ventilated). The comparison patient temperature source was predominantly esophageal $(n=10)$ followed by bladder $(n=5)$ or rectal $(n=5)$. There were a total of $999 \mathrm{~h}$ of paired patient temperature data from esophageal (50\%), bladder (25\%), and rectal (25\%) temperatures. Bland-Altman analysis demonstrated good agreement with the superficial temperature monitor and core temperature measures in all patients overall, with a difference mean of $0.06 \pm 0.39 C(P=0.99)$ and no proportional bias noted $(\beta=0.002$, $P=0.917)$.

Conclusions: Continuous noninvasive temperature monitoring is a suitable alternative method for assessing core temperature during TTM. Future studies should focus on developing connectivity with a continuous noninvasive temperature monitor to approximate core temperature during TTM.

Keywords: Normothermia, Hypothermia, Targeted temperature management, Core temperature, Continuous noninvasive temperature monitoring

${ }^{*}$ Correspondence: MICURN32@gmail.com

${ }^{1}$ Neurocritical Care Advanced Practice Providers, Neuro Intensive Care Unit, University of Maryland Medical Center, 22 South Greene Street, Baltimore, MD 21201, USA

Full list of author information is available at the end of the article

\section{Introduction}

The detrimental effects of hyperthermia in the neurologically injured patient, along with the beneficial effects of normothermia, have been widely studied in the critically ill neurological population [1-3]. For these reasons, 
targeted temperature management (TTM) has become a mainstay therapy in this population.

During TTM, multiple sites are utilized for continuous core temperature monitoring, including bladder, esophageal, and rectal probes. The Neurocritical Care Society (NCS) TTM guideline suggests that esophageal and bladder probes most accurately reflect temperatures of pulmonary artery catheters and recommend using esophageal temperature probes during TTM [1]. Esophageal probe use is limited to intubated patients, and there are increased demands to move away from bladder temperature monitoring in order to prevent catheter-associated urinary tract infections. Rectal probes are the least accurate, and temporal artery probes were not recommended for TTM because they were not accurate and did not have continuous monitoring capabilities [1].

The purpose of this study was to determine whether a continuous noninvasive temperature monitoring system using a continuous temporal artery probe is as accurate as the current invasive measures during TTM.

\section{Methods}

\section{Patient Selection}

This study was a prospective observational study of a continuous temperature monitor during TTM. Data collection for this study was approved under an existing IRB registry for all patients admitted to our neurocritical care unit (NCCU). All adult patients undergoing TTM via a gel pad surface temperature modulating device (TMD; Arctic-Sun 5000 Temperature Management System, C.R. Bard Inc., Louisville, $\mathrm{CO}$ ) and an existing core temperature monitoring device (bladder, rectal, or esophageal) were eligible for the study.

\section{Target Temperature Management and Temperature Monitoring}

The noninvasive temperature monitoring system $\left(3 \mathrm{M}^{\mathrm{TM}}\right.$ Bair Hugger $^{\mathrm{TM}}$ Temperature Monitoring System, St Paul, $\mathrm{MN}$ ) allows continuous monitoring through a sensor placed on the forehead. The prototype device was initially studied in a non-emergent cardiac surgery population where it demonstrated agreement with the golden standard Pulmonary Artery (PA) catheter core temperature readings [4].

The nursing staff and advanced practice providers were in-serviced on use of the continuous noninvasive temperature monitoring system $\left(3 \mathrm{M}^{\mathrm{TM}}\right.$ Bair Hugger ${ }^{\mathrm{TM}}$ Temperature Monitoring System, St Paul, MN) prior to initiation of use in the NCCU. Education included, but was not limited to application/removal of sensor, operation/trouble shooting of the control unit, safety monitoring (e.g., skin break down under sensor), and contraindications of use (e.g., existing skin breakdown). The sensor was placed on the lateral forehead and replaced on the contralateral side every $24 \mathrm{~h}$ per manufacturer recommendations and/ or when the sensor was removed for imaging or the sensor became dislodged such as with profuse diaphoresis [5].

Our institution TTM protocol encompasses the use of TMDs for controlled normothermia and induced hypothermia. Patients undergoing normothermia (goal $37{ }^{\circ} \mathrm{C}$ ) or hypothermia post-cardiac arrest (goal $32-34{ }^{\circ} \mathrm{C}$ prior to rewarming) were included in the study. Choice of TMD and location of temperature monitoring was left to the discretion of the clinical team, with the following notable exceptions. Esophageal temperature probes (Smiths Medical, Minneapolis, MN) were utilized in orally intubated patients who were not utilizing esophageal cooling devices (ECDs-Attune Medical, Chicago, IL). Bladder temperature probes (Bard, Covington, GA) were utilized in patients when medically indicated (e.g., retention, decubitus ulcer). Rectal temperature probes (Stryker Medical, Portage, MI) were utilized in nonintubated patients or patients where foley catheter was not indicated or could not be replaced with temperature sensing foley catheter for medical reasons (e.g., Benign prostatic hyperplasia, prior traumatic insertion, coude foley catheter). Nursing assessment and documentation included hourly neurological exams, vital signs, Intracranial Pressure, core temperature, temporal temperature, and machine water temperature. Skin integrity was assessed every $4 \mathrm{~h}$ for rash or evidence of breakdown from gel pads. Shivering was assessed and treated every hour using the bedside shivering assessment scale (BSAS) as previously described $[6,7]$.

Once a patient was determined eligible for the study, a disposable skin temperature probe $\left(3 \mathrm{M}^{\mathrm{TM}}\right.$ Bair Hugger ${ }^{\mathrm{TM}}$ Temperature Monitoring System, St Paul MN, Sensor model 360) was placed on the lateral forehead above the eyebrow. The probe was then connected to the monitoring device $\left(3 \mathrm{M}^{\mathrm{TM}}\right.$ Bair Hugger ${ }^{\mathrm{TM}}$ Temperature Monitoring System Control Unit, 120 V), which was interfaced with the bedside monitor. The core temperature monitoring device was connected to the TMD and also interfaced with the bedside monitor.

\section{Data Collection}

Baseline characteristics collected on patients undergoing TTM included height, weight, BMI, age, gender, diagnosis, temperature source, and target temperature. Standard anti-shivering interventions were utilized during TTM therapy [8]. Due to concerns that shivering may affect the efficacy of the continuous noninvasive temperature monitoring system, we collected data on shivering interventions including bedside shivering 
assessment score (BSAS), and any anti-shivering medications the patients received during TTM.

Core and noninvasive surface temperatures were simultaneously recorded for up to $72 \mathrm{~h}$ of TTM. Continuous temperature data were collected via BedMaster $^{\circledR}$ (Excel Medical Electronics Inc., Jupiter, FL) vital signs collection system beginning at the time of TTM initiation. The two sets of temperature data were downloaded from the clinical data acquisition storage system.

\section{Statistical Analysis}

Our primary outcome was agreement between the esophageal, bladder, or rectal temperatures and the continuous noninvasive temperature monitor temperatures with a difference of $\leq 0.5{ }^{\circ} \mathrm{C}$. As in other studies, we defined $0.5 \mathrm{C}$ a priori as an acceptable limit of agreement [9-11]. These limits reflect the normal magnitude of human circadian temperature variation [12, 13]. We performed a Bland-Altman analysis to evaluate agreement between the noninvasive device and each of the invasive temperature monitoring devices individually (rectal, bladder, and esophageal), assuming that the true value changes from measurement to measurement [14]. In a Bland-Altman analysis, the bias and 95\% limits of agreement are determined. The bias, which is estimated by the average difference, summarizes the lack of agreement between the two methods, whereas the 95\% limits of agreement represent where $95 \%$ of future differences between the two methods would be expected. We calculated bias (esophageal temperature minus double-sensor temperature or bladder temperature minus double-sensor temperature or rectal temperature minus continuous noninvasive temperature) for each outcome and for each time point, and we also calculated the mean bias across all pairs of measurements. We created a Bland-Altman plot of bias versus the average of each pair of measurements for visual representation of the agreement with the standard or reference core temperature measurements over the range of temperatures. For the entire cohort and additionally for each site of temperature assessment, proportional bias was assessed. This was done by performing a linear regression whereby the dependent variable was the difference between two measures and the independent variable was mean temperature for each paired measurement. Regressions with a statistically significant result $(P<0.05)$ were considered to demonstrate proportional bias. All analyses were performed with SPSS statistical software (IBM Corp. Released 2019. IBM SPSS Statistics for Windows, Version 26.0. Armonk, NY: IBM Corp.)

\section{Results}

Clinical Characteristics (Table 1)

There were $999 \mathrm{~h}$ of paired temperature recordings done on twenty subjects between January 2018 and May 2018. Only one subject was targeted to hypothermia (33 C), with the rest $(n=19,95 \%)$ targeted to normothermic temperature goals (36-37 C). Esophageal temperature was the most common source $(n=10,50 \%)$ with an average duration of $55.7 \pm 17.9 \mathrm{~h}$, followed by rectal temperature $(n=5,25 \%)$ with an average duration of $51.4 \pm 17.2 \mathrm{~h}$ and bladder temperature $(n=5,25 \%)$ with an average duration of $37 \pm 21.9 \mathrm{~h}$.

All subjects were endotracheally intubated during the study period. No reports of skin irritation or breakdown were reported at the skin temperature measurement sites.

Shivering was well controlled (BSAS $\leq 1$ ) in $70 \%$ of the subjects throughout the duration of cooling with moderate use of analgosedation. Paralytics were utilized in two patients: one patient as part of rapid sequence intubation and for ventilator synchrony in another patient with acute lung injury (Table 2).

\section{Bland-Altman Analysis of Temperature Measurements (Table 3, Fig. 1)}

The majority of the paired measurements were done in the range of $36.0-38.2{ }^{\circ} \mathrm{C}(n=759 \mathrm{~h}, 76 \%)$, with a minority done below $36.0{ }^{\circ} \mathrm{C}(n=230,23 \%)$ and very few $\geq 38.3{ }^{\circ} \mathrm{C}(n=10,1 \%)$. No difference in agreement was noted with moderate or severe shivering, though

\section{Table 1 Baseline characteristics}

\begin{tabular}{lr}
\hline Characteristics & $\mathbf{N}=\mathbf{2 0}$ \\
\hline Body mass index $\left(\mathrm{kg} / \mathrm{m}^{2}\right)$ & $28.9(9.8)$ \\
Age (years) & $57.1(14)$ \\
Female & $11(55)$ \\
Diagnosis & \\
Subarachnoid hemorrhage & $6(30)$ \\
Intracerebral hemorrhage & $7(35)$ \\
Seizure & $2(10)$ \\
Cardiac arrest & $3(15)$ \\
\hline Ischemic stroke & $2(10)$ \\
\hline Target temperature $\left({ }^{\circ} \mathrm{C}\right)$ & \\
\hline $33^{\circ} \mathrm{C}$ & $1(5)$ \\
$36^{\circ} \mathrm{C}$ & $1(5)$ \\
$37^{\circ} \mathrm{C}$ & $18(90)$ \\
\hline Temperature $(\mathrm{T} 1)$ source & $10(50)$ \\
\hline Esophageal & $5(25)$ \\
\hline Bladder & $5(25)$ \\
\hline Rectal &
\end{tabular}

Continuous data shown as mean (SD), categorical data as $n(\%)$ 
Table 2 Shivering characteristics during TTM

\begin{tabular}{|c|c|c|}
\hline Characteristic & $N=20$ & Percent \\
\hline \multicolumn{3}{|l|}{ Shivering (h) } \\
\hline$B S A S=0$ & 8 & 40 \\
\hline $\mathrm{BSAS}=0-1$ & 6 & 30 \\
\hline$B S A S=0-2$ & 5 & 25 \\
\hline$B S A S=0-3$ & 1 & 5 \\
\hline Medications & $N=20$ & Mean (range)/24 h \\
\hline Propofol (mcg/kg/min) & $7(35)$ & $27(0-50)$ \\
\hline Dexmedetomidine (mcg/kg/h) & $7(35)$ & $0.6(0-1.5)$ \\
\hline Fentanyl (mcg/h) & $3(15)$ & $94(0-200)$ \\
\hline Morphine (mg/h) & $2(10)$ & $4.5(0-8)$ \\
\hline Meperidine (mg) & $10(50)$ & $39(13-100)$ \\
\hline Rocuronium (mg) & $2(10)$ & $60(50-70)$ \\
\hline Cisatracurium (mcg/kg/min) ${ }^{b}$ & $1(5)$ & $0.4(0-1.25)$ \\
\hline Dantrolene (mg) & $1(5)$ & $80(0-80)$ \\
\hline
\end{tabular}

BSAS bedside shivering assessment scale

a Neuromuscular blockade agents used for endotracheal intubation $(n=1)$ and for management of ARDS $(n=1)$

b Cisatracurium used for management of ARDS

Table 3 Temperature measurements

\begin{tabular}{llll} 
Measurements & Esophageal & \multicolumn{2}{l}{$\begin{array}{l}\text { Core temperature } \\
\text { measurement sites }\end{array}$} \\
\cline { 3 - 4 } & & Bladder & Rectal \\
\hline Temperature range $\left({ }^{\circ} \mathrm{C}\right)$ & $31.5-38.6$ & $34.8-37.8$ & $35.2-37.8$ \\
Difference mean $\pm \mathrm{SD}$ & $0.31 \pm 0.54$ & $0.2 \pm 0.4$ & $0.16 \pm 0.38$ \\
Significance & $P=1.00$ & $P=1.00$ & $P=1.00$ \\
Linear regression $\beta$ & -0.01 & 0.068 & 0.782 \\
Significance & $P=0.507$ & $P=0.342$ & $P<0.001$ \\
\hline
\end{tabular}

there was very little moderate or severe shivering $(\mathrm{BSAS}>1)$ noted during the study period.

There was good agreement with the esophageal core temperature with no proportional bias $(P=0.507)$, though as apparent in Fig. 1a, there were some measurements in the range of 36.0-38.0 that were outside the $95 \%$ boundaries. There was overall greater agreement in the hypothermic range $(<36.0 \mathrm{C})$. Bladder temperature comparisons showed good agreement with no proportional bias $(P=0.342)$ across the range of temperatures (Table 3, Fig. 1b). The paired measurements with rectal temperature demonstrated a proportional bias $(P<0.001)$, despite a small difference between measures (Table 3, Fig. 1c).

\section{Discussion}

In this study of patients undergoing TTM, we evaluated a continuous noninvasive temperature monitor against currently utilized invasive core temperature monitoring devices including bladder temperature probes, rectal temperature probes, and esophageal temperature probes. To our knowledge, this is the first comparative analysis of a continuous noninvasive temperature monitor to invasive core temperature measures in a population undergoing prolonged TTM. The results demonstrate that the continuous noninvasive temperature monitor correlates with bladder and esophageal temperature measurements. Proportional correlation was identified between the continuous noninvasive temperature monitor sensor and the rectal temperature. This may be due to the known lag time between rectal temperature and core temperature and the effects of feces and inflammation on temperature recordings [15-20].

TTM improves outcomes by reducing effects of the primary insult and minimizing the secondary neurological insult often associated with hypoxic/ischemic encephalopathy (HIE) and intracranial hypertension [1-3, 21]. Ideally, all patients undergoing TTM should have continuous core temperature measurements. The surface overlying the temporal artery is an ideal location for noninvasive continuous core temperature monitoring because the internal carotid artery supplies it and it is a short distance from the aorta [20, 22]. The temporal artery is void of mucous membranes and has little to no arteriovenous anastomoses, providing a continuous blood flow free of vasomotor control that could potentially alter the surface temperature [i.e., vasoconstriction] [20]. In order for the temporal surface temperature to 


\section{A Esophageal Temperature $(\mathrm{N}=10)$}

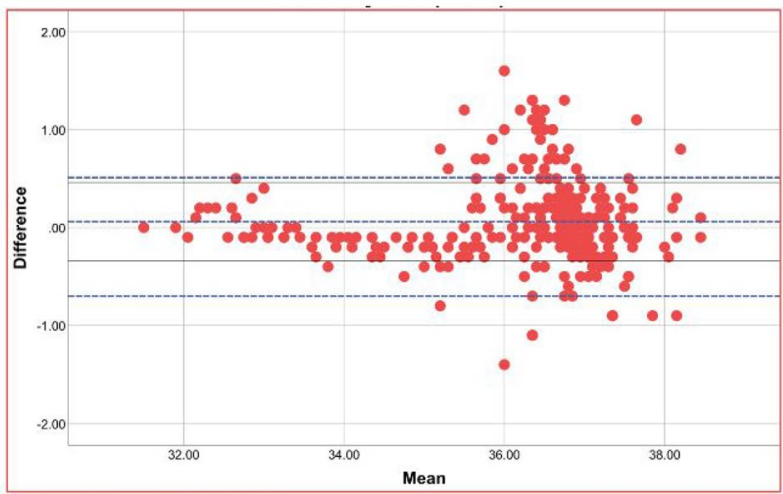

\section{Rectal Temperature $(\mathrm{N}=5)$}

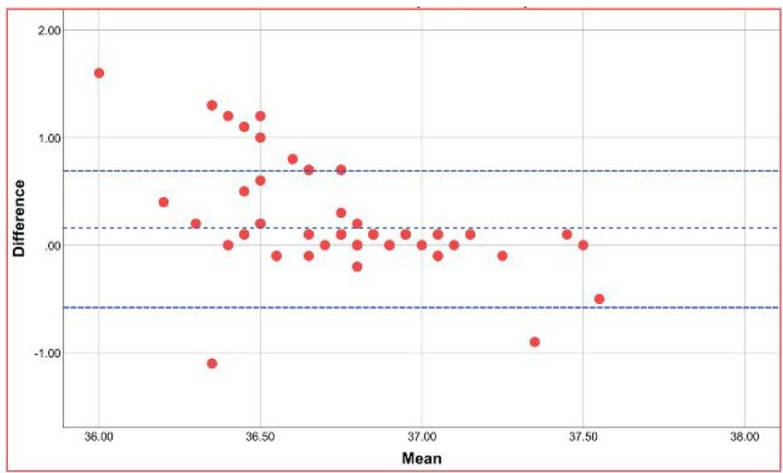

B Bladder Temperature $(\mathrm{N}=5)$

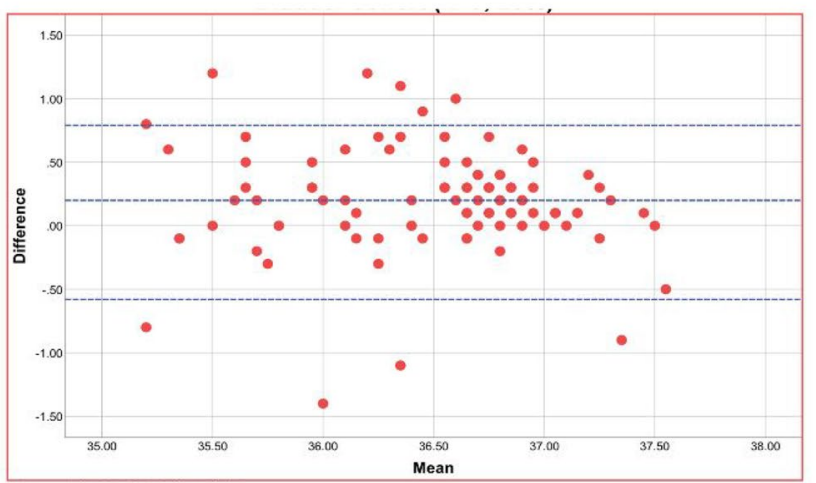

Fig. 1 Comparison of paired temperature measurements. Plot comparing paired mean ( $x$-axis) with difference in mean ( $y$-axis). Hashed lines represent population mean and $95 \% \mathrm{Cl}$. All temperature measurements reported as ${ }^{\circ} \mathrm{C}$

reflect the temporal artery temperature, thermal heat loss must be eliminated [20, 23]. The device used in this study $\left(3 \mathrm{M}^{\mathrm{TM}}\right.$ Bair Hugger ${ }^{\mathrm{TM}}$ Temperature Monitoring System, St Paul MN, Sensor model 360) provides an isothermal pathway via a single use sensor placed on the forehead. This isothermal pathway is created as the sensor develops a zone of insulation that eliminates heat loss, thereby bringing the core temperature to the skin surface where it is continuously recorded and displayed [5, 24].

There has been extensive research comparing multiple methods of core temperature monitoring. Heat is balanced between the peripheral and core compartments of the body. The core compartment comprises approximately $50 \%$ of the body mass and consists of the wellperfused deep tissues and organs of the trunk and head [25]. The temperature of the core compartment remains consistent making it the ideal source for continuous temperature monitoring. The pulmonary artery catheter has long been recognized as the gold standard core temperature monitoring site; however, it is highly invasive, and placement/maintenance is not without significant cardiac risks $[1,16,19,22,23,26]$. Our study demonstrated accuracy between the continuous noninvasive temperature monitor and commonly used surrogates for PA catheter-bladder and esophageal probes. Bladder temperatures have been found to be reliable when compared to PA catheters $[1,16,27]$, but require placement of a foley catheter, placing patients at risk for catheter-associated urinary tract infections (CAUTI). Temperatures obtained from bladder monitoring can be erroneous in patients with anuria/oliguria and can lag behind core temperatures during rapid core temperature fluctuations $[16,19$, $24,28]$. Esophageal monitoring is also considered a reliable site for core temperature monitoring; however, its use is limited to patients who are orally intubated [1, 16, 21, $22,24,26]$. Additionally, administration of medications and fluids can transiently falsely alter the temperature in the esophagus [16].

There are several limitations to the study that warrant discussion. Our study size was relatively small $(n=20)$, 
but it is comparable to other studies that compared temperature monitoring systems [19, 24, 26, 28]. However, the amount of data (i.e., hourly temperature measurements for up to $72 \mathrm{~h}$ ) is far greater than what other studies utilized [17, 18, 23, 24, 26, 28]. Despite the patient size, there was good agreement between the continuous noninvasive temperature monitor, the bladder temperature probe, and esophageal temperature probe demonstrated with the Bland-Altman analyses. We were unable to determine the accuracy of the continuous noninvasive temperature monitor in patients with spontaneous temperature fluctuations although the patients in the study did have core temperature fluctuations, while on TTM, therefore one could confer that it is comparable. Finally, some might view $0.2-0.3 \mathrm{C}$ deviation of the esophageal and bladder temperature probes as too high of a deviation; however, all previous core temperature monitors, including bladder and esophageal temperature measurement devices, have commonly utilized a higher threshold of $0.3-0.5 \mathrm{C}[9,11,16,21-24,26,28]$. The major strength of the study was the continuous recording of both sets of temperatures into a clinical data acquisition storage system, allowing for simultaneous real-time comparison.

\section{Conclusions}

Based on the data collected, the continuous noninvasive temperature monitor $\left(3 \mathrm{M}^{\mathrm{TM}}\right.$ Bair Hugger ${ }^{\mathrm{TM}}$ Temperature Monitoring System, St Paul, MN) device was in good agreement with both esophageal and bladder temperatures and should be considered for use as the primary source of temperature monitoring in patients undergoing TTM. Future studies should focus on developing connectivity with a continuous noninvasive temperature monitor as a suitable alternative to approximate core temperature during TTM.

\footnotetext{
Author details

${ }^{1}$ Neurocritical Care Advanced Practice Providers, Neuro Intensive Care Unit, University of Maryland Medical Center, 22 South Greene Street, Baltimore, MD 21201, USA. ${ }^{2}$ Zucker School of Medicine, Hofstra/Northwell Health, Hempstead, USA. ${ }^{3}$ Department of Nursing, University of Maryland Medical Center, Baltimore, USA. ${ }^{4}$ Department of Nursing, Hood College, Frederick, USA. ${ }^{5}$ Program in Trauma, Department of Neurology, University of Maryland School of Medicine, Baltimore, USA.
}

\section{Author contributions}

Authorship requirements have been met and the final manuscript was approved by all authors.

\section{Source of support}

$3 \mathrm{M}^{\mathrm{TM}}$ St Paul, MN, provided SPOT ON disposable sensors at no cost for the study but had no other involvement in the conduct of the study or analysis of research data.

\section{Conflict of interest}

Dr N. Badjatia has received research grants from Maryland Industrial Partnerships, outside of the submitted work. All other authors declare that they have no conflicts of interest.
Ethical approval

IRB approval not applicable. Ethical standards were adhered to.

\section{Publisher's Note}

Springer Nature remains neutral with regard to jurisdictional claims in published maps and institutional affiliations.

Published online: 6 July 2020

\section{References}

1. Madden LK, et al. The implementation of targeted temperature management: an evidence-based guideline from the Neurocritical Care Society. Neurocrit Care. 2017;27(3):468-87. https://doi.org/10.1007/s1202 8-017-0469-5.

2. Tripathy S, Mahapatra A. Targeted temperature management in brain protection: an evidence-based review. Indian J Anaesth. 2015;59(1):9-14. https://doi.org/10.4103/0019-5049.149442.

3. Muengtaweepongsa S, Srivilaithon W. Targeted temperature management in neurological intensive care unit. World J Methodol. 2017;7(2):55. https://doi.org/10.5662/wjm.v7.i2.55.

4. Eshraghi Y, Sessler DI. Exploratory method-comparison evaluation of a disposable non-invasive zero heat flow thermometry system. American Society of Anesthesiologists, https://www.asaabstracts.com/strands/asaab stracts/abstract.htm?year $=2012 \&$ index $=8 \&$ absnum $=4738$. Accessed 25 May 2020.

5. 3M: SpotOn temperature monitoring system model 370 , brochure. https ://multimedia.3m.com/mws/media/8798030/operators-manual-engli sh.pdf. Accessed 5 September 2019

6. Badjatia N, et al. Metabolic impact of shivering during therapeutic temperature modulation. Stroke. 2008;39(12):3242-7. https://doi. org/10.1161/strokeaha.108.523654.

7. Khan I, et al. Esophageal cooling device versus other temperature modulation devices for therapeutic normothermia in subarachnoid and intracranial hemorrhage. Ther Hypoth Temp Manag. 2018;8(1):53-8. https //doi.org/10.1089/ther.2017.0033.

8. Choi H Alex, et al. Prevention of shivering during therapeutic temperature modulation: the Columbia anti-shivering protocol. Neurocrit Care. 2011;14(3):389-94. https://doi.org/10.1007/s12028-010-9474-7.

9. Suleman MI, Doufas AG, Akca O, Ducharme M, Sessler DI. Insufficiency in a new temporal-artery thermometer for adult and pediatric patients. Anesth Analg. 2002;95:67-71.

10. Moran JL, Peter JV, Solomon PJ, et al. Tympanic temperature measurements: are they reliable in the critically ill? A clinical study of measures of agreement. Crit Care Med. 2007;35:155-64

11. Kimberger $\mathrm{O}$, Cohen $\mathrm{D}$, Illievich $U$, Lenhardt R. Temporal artery versus bladder thermometry during perioperative and intensive care unit monitoring. Anesth Analg. 2007;105:1042-7.

12. Tayefeh F, Plattner O, Sessler DI, Ikeda T, Marder D. Circadian changes in the sweating-to-vasoconstriction interthreshold range. Pflugers Arch. 1998;435:402-6.

13. Sessler DI, Lee KA, McGuire J. Isoflurane anesthesia and circadian temperature cycles in humans. Anesthesiology. 1991;75:985-9.

14. Bland JM, Altman DG. Statistical methods for assessing agreement between two methods of clinical measurement. Lancet. 1986;1:307-10.

15. Platt M, Vicario S. 141/Heat illness. Rosen's emergency medicine, vol. 2. 8th ed. Philadelphia: Saunders; 2009. p. 1904.

16. Chacko B, Peter J. Temperature monitoring in the intensive care unit. Indian J Respir Care. 2018;7(1):28. https://doi.org/10.4103/ijrc.ijrc_13_17.

17. Greenes D, Fleisher G. When body temperature changes, does rectal temperature lag? J Pediatr. 2004;144(6):824-6. https://doi.org/10.1016/ s0022-3476(04)00167-2.

18. Carr EA, et al. Comparison of temporal artery to rectal temperature measurements in children Up to 24 months. J Pediatr Nurs. 2011;26(3):179-85. https://doi.org/10.1016/j.pedn.2009.12.072.

19. Shin J, et al. Core temperature measurement in therapeutic hypothermia according to different phases: comparison of bladder, rectal, 
and tympanic versus pulmonary artery methods. Resuscitation. 2013;84(6):810-7. https://doi.org/10.1016/j.resuscitation.2012.12.023.

20. Exergen. Temperature Assessment via the Temporal Artery: Validation... https://www.exergen.com/wp-content/uploads/2016/03/Pompei-M.1999-Temperature-assessment-via-the-TA-Validation.pdf. Accessed 6 September 2019.

21. Dahyot-Fizelier C, et al. Accuracy of zero-heat-flux cutaneous temperature in intensive care adults. Crit Care Med. 2017;45(7):e715-7. https://doi. org/10.1097/ccm.00000000000002317.

22. Hooper VD. Accuracy of noninvasive core temperature measurement in acutely ill adults: the state of the science. Biol Res Nurs. 2006;8(1):24-34. https://doi.org/10.1177/1099800406289151.

23. Furlong $D$, et al. Comparison of temporal to pulmonary artery temperature in febrile patients. Dimens Crit Care Nurs. 2015;34(1):47-52. https:// doi.org/10.1097/dcc.00000000000000090.

24. Mäkinen M-T, et al. Novel zero-heat-flux deep body temperature measurement in lower extremity vascular and cardiac surgery. J Cardiothorac Vasc Anesth. 2016;30(4):973-8. https://doi.org/10.1053/j.jvca.2016.03.141.
25. Sessler. Perioperative thermoregulation and heat balance-dashboard. http://wiki.med.uottawa.ca/download/attachments/7438400/Sessl er_Periop_ThermoregulatHeat_Balance.pdf?version $=1$ \&modificationDat e=1322942999000. Accessed 7 September 2019.

26. Teunissen LPJ, et al. Non-invasive continuous core temperature measurement by zero heat flux. Physiol Meas. 2011;32(5):559-70. https://doi. org/10.1088/0967-3334/32/5/005

27. Knapik P, et al. Relationship between blood, nasopharyngeal and urinary bladder temperature during intravascular cooling for therapeutic hypothermia after cardiac arrest. Resuscitation. 2012;83(2):208-12. https://doi. org/10.1016/j.resuscitation.2011.09.001.

28. Langham GE, et al. Noninvasive temperature monitoring in postanesthesia care units. Anesthesiology. 2009;111(1):90-6. https://doi.org/10.1097/ aln.0b013e3181a864ca. 